\title{
Parametrically tunable soliton-induced resonant radiation by three-wave mixing
}

Zhou, Binbin; Liu, Xing; Guo, Hairun; Zeng, Xianglong; Chen, X. F.; Chung, Hung-ping ; Chen, Yen-Hung ; Bache, Morten

Published in:

Physical Review Letters

Link to article, DOI:

10.1103/PhysRevLett.118.143901

Publication date:

2017

Document Version

Publisher's PDF, also known as Version of record

Link back to DTU Orbit

Citation (APA):

Zhou, B., Liu, X., Guo, H., Zeng, X., Chen, X. F., Chung, H., Chen, Y-H., \& Bache, M. (2017). Parametrically tunable soliton-induced resonant radiation by three-wave mixing. Physical Review Letters, 118(14), [143901]. https://doi.org/10.1103/PhysRevLett.118.143901

\section{General rights}

Copyright and moral rights for the publications made accessible in the public portal are retained by the authors and/or other copyright owners and it is a condition of accessing publications that users recognise and abide by the legal requirements associated with these rights.

- Users may download and print one copy of any publication from the public portal for the purpose of private study or research.

- You may not further distribute the material or use it for any profit-making activity or commercial gain

- You may freely distribute the URL identifying the publication in the public portal 


\title{
Parametrically Tunable Soliton-Induced Resonant Radiation by Three-Wave Mixing
}

\author{
B. B. Zhou, ${ }^{1}$ X. Liu, ${ }^{1}$ H. R. Guo, ${ }^{1, \dagger}$ X. L. Zeng,,${ }^{1,}$ X. F. Chen, ${ }^{2}$ H. P. Chung, ${ }^{3}$ \\ Y. H. Chen, ${ }^{3}$ and M. Bache ${ }^{1, *}$ \\ ${ }^{1}$ DTU Fotonik, Technical University of Denmark, DK-2800 Kgs. Lyngby, Denmark \\ ${ }^{2}$ Department of Physics and Astronomy, Shanghai Jiao Tong University, Shanghai 200240, China \\ ${ }^{3}$ Department of Optics and Photonics, National Central University, Jhongli 320, Taiwan
}

(Received 30 June 2016; published 5 April 2017)

\begin{abstract}
We show that a temporal soliton can induce resonant radiation by three-wave mixing nonlinearities. This constitutes a new class of resonant radiation whose spectral positions are parametrically tunable. The experimental verification is done in a periodically poled lithium niobate crystal, where a femtosecond near-IR soliton is excited and resonant radiation waves are observed exactly at the calculated soliton phasematching wavelengths via the sum- and difference-frequency generation nonlinearities. This extends the supercontinuum bandwidth well into the mid IR to span $550-5000 \mathrm{~nm}$, and the mid-IR edge is parametrically tunable over $1000 \mathrm{~nm}$ by changing the three-wave mixing phase-matching condition. The results are important for the bright and broadband supercontinuum generation and for the frequency comb generation in quadratic nonlinear microresonators.
\end{abstract}

DOI: 10.1103/PhysRevLett.118.143901

The temporal optical soliton is quite remarkable: while often desired because it retains its form despite dispersive effects [1], perturbing the perfect solitary shape may also lead to phase matching of a so-called resonant radiation (RR) wave [2] (also known as soliton-induced optical Cherenkov radiation [3]). RR waves are today considered a coherent source of laser radiation [4], in particular for the supercontinuum generation [5] where they contribute coherently for extending the supercontinuum bandwidth, as well as in the ultrashort pulse generation in the UV and the mid IR [6-9]. Traditionally, RR waves are generated by four-wave mixing (4WM) through the Kerr self-phase modulation (SPM) term $|A|^{2} A$ [10-12]. Recently, other nonstandard $4 \mathrm{WM}$ terms were shown to support RR waves, namely the conjugate SPM term $|A|^{2} A^{*}$ [13-16] and the third-harmonic generation term $A^{3}[17,18]$.

Phase-mismatched (cascaded) three-wave mixing (3WM) in quadratic nonlinear crystals can generate a negative selfdefocusing Kerr-like nonlinearity [19,20], and when a temporal soliton is excited [21], this gives octave-spanning supercontinua [22-25] that are filament free [26-29]. So far, only RR waves generated by the cascaded self-defocusing SPM effect have been verified [27-29]. In this Letter, we show that a new class of RR waves exist in quadratic nonlinear crystals, induced by the soliton through the $3 \mathrm{WM}$ processes sum-frequency generation (SFG, $A^{2}$ ) and difference-frequency generation $\left(\mathrm{DFG}, A^{*} A\right)$. These $\mathrm{RR}$ waves contribute coherently to the supercontinuum, making it brighter and more broadband, and remarkably, their center wavelengths are parametrically tunable by adjusting only the $3 \mathrm{WM}$ phase-matching conditions, giving an additional control over the supercontinuum, often lacking in the 4WM RR case. Here, we show direct experimental proof of $3 \mathrm{WM}$
RR waves excited by an infrared (IR) soliton in a periodically poled lithium niobate (PPLN) crystal (Fig. 1). The SFG and DFG RR waves are observed, and we demonstrate how their center wavelengths can be strongly tuned by changing the $3 \mathrm{WM}$ phase-matching conditions. The DFG RR wave in particular provides a tunable extension of the supercontinuum well into the mid IR $(4.0-5.5 \mu \mathrm{m}$ range, important for ultrafast vibrational spectroscopy).

In order to describe these novel RR waves, the nonlinear terms cannot be truncated, which implies resolving the electric field on a carrier level. We use the nonlinear analytic envelope equation [17,30], whose formalism conveniently

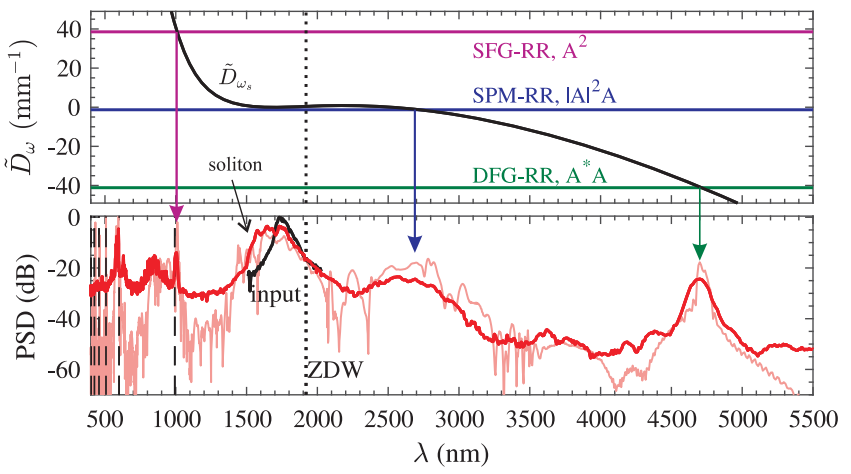

FIG. 1. Supercontinuum recorded for $\lambda_{0}=1.75 \mu \mathrm{m}$ and $I_{0}=150$, using a $10 \mathrm{~mm}$ PPLN with $\Lambda=30.0 \mu \mathrm{m}$; the experimental data (thick red) are directly compared to a numerical simulation (thin light red) and calculated higher-order quasiphase matching resonances (dashed lines). The power-spectral densities (PSDs) are normalized to the peak input PSD so that the average output power matches the input power. The top plot shows the theoretical RR phase-matching conditions to the soliton using $\lambda_{s}=1.68 \mu \mathrm{m}$. ZDW: zero-dispersion wavelength $(1.92 \mu \mathrm{m})$. 
retains an envelopelike equation. The $\chi^{(2)}-\chi^{(3)}$ nonlinear dynamics at the pump frequency $\omega_{1}$ is described in a single equation of the $e$-polarized pump envelope $A$ in the comoving reference frame $(\zeta, \tau)$ [31-33],

$$
\begin{aligned}
& i \partial_{\zeta} A+\hat{D}_{\omega_{1}} A \\
& =-\kappa^{(2)}\left[\frac{1}{2} A^{2} e^{-i \omega_{1} \tau-i \Delta_{\mathrm{pg}} \zeta}+A^{*} A e^{i \omega_{1} \tau+i \Delta_{\mathrm{pg}} \zeta}\right]_{+} \\
& -\kappa^{(3)}\left[|A|^{2} A+|A|^{2} A^{*} e^{i 2 \omega_{1} \tau+i 2 \Delta_{\mathrm{pg}} \zeta}+\frac{1}{3} A^{3} e^{-i 2 \omega_{1} \tau-i 2 \Delta_{\mathrm{pg} \zeta}}\right]_{+} .
\end{aligned}
$$

Self-steepening terms and delayed Raman effects [31] are here neglected as they do not influence the following phasematching analysis. The nonlinear parameters are $\kappa^{(2)} \propto \chi^{(2)}$ and $\kappa^{(3)} \propto \chi^{(3)}$, related to quadratic and cubic nonlinear terms, respectively. The operator, $\hat{D}_{\omega_{1}}=\sum_{m=2} m !^{-1} k_{m}\left(\omega_{1}\right)$ $\left(i \partial_{\tau}\right)^{m}$, accounts for dispersion in the time domain, where $k_{m}(\omega)=d^{m} k(\omega) / d \omega^{m}$ are the higher-order dispersion coefficients. The dispersion is conveniently evaluated exactly in the frequency domain as $\tilde{D}_{\omega_{1}}(\omega)=k(\omega)-k_{1}\left(\omega_{1}\right)$ $\left(\omega-\omega_{1}\right)-k\left(\omega_{1}\right)$. The constant term, $\Delta_{\mathrm{pg}}=\omega_{1} k_{1}\left(\omega_{1}\right)-$ $k\left(\omega_{1}\right)=\omega_{1}\left(1 / v_{g}-1 / v_{p}\right)$, accounts for the phase-groupvelocity mismatch (carrier-envelope phase slip), where $v_{g}=1 / k_{1}\left(\omega_{1}\right)$ is the pump group velocity, and $v_{p}=$ $c / n\left(\omega_{1}\right)$ is the pump phase velocity. Finally, the + sign implies that only the positive frequency content of the nonlinear term is used [30].

The equations will support a number of RR phasematching conditions between a soliton at frequency $\omega_{s}$ (without loss of generality, we can take $\omega_{s}=\omega_{1}$ ) and a "dispersive" (i.e., nonsolitonic) RR wave,

$$
\begin{aligned}
& \tilde{D}_{\omega_{s}}\left(\omega_{\mathrm{RR}}\right)=q_{s}, \quad\left(\mathrm{SPM}-\mathrm{RR},|A|^{2} A\right), \\
& \tilde{D}_{\omega_{s}}\left(\omega_{\mathrm{RR}}\right)=-q_{s}+2 \Delta_{\mathrm{pg}}, \quad\left(\mathrm{cSPM}-\mathrm{RR},|A|^{2} A^{*}\right), \\
& \tilde{D}_{\omega_{s}}\left(\omega_{\mathrm{RR}}\right)=3 q_{s}-2 \Delta_{\mathrm{pg}}, \quad\left(\mathrm{THG}-\mathrm{RR}, A^{3}\right), \\
& \tilde{D}_{\omega_{s}}\left(\omega_{\mathrm{RR}}\right)=2 q_{s}-\Delta_{\mathrm{pg}}, \quad\left(\mathrm{SFG}-\mathrm{RR}, A^{2}\right), \\
& \tilde{D}_{\omega_{s}}\left(\omega_{\mathrm{RR}}\right)=\Delta_{\mathrm{pg}}, \quad\left(\mathrm{DFG}-\mathrm{RR}, A^{*} A\right) .
\end{aligned}
$$

These were found by inserting the ansatz, $A(\zeta, \tau)=$ $F_{s}(\tau) e^{i q_{s} \zeta}+g(\zeta, \tau)$ [41], into Eq. (1) [33]. Here, $F_{s}(\tau) e^{i q_{s} \zeta}$ is the exact nonlinear solution when taking into account only the SPM nonlinearity and GVD, $F_{s}$ is the soliton envelope, $q_{s}$ is the soliton nonlinear wave number, and $g$ is the RR wave [33]. The first phasematching condition is the traditional RR induced by SPM, the second is the RR from the "conjugate SPM" term (cSPM-RR, also known as negative-frequency RR), while the third is the "third-harmonic generation RR" or simply THG-RR. The last two are the new $3 \mathrm{WM}$ phase-matching conditions from the SFG and DFG $\chi^{(2)}$ nonlinear terms, here, presented for the first time. In quadratic nonlinear crystals, the Kerr-like SPM-RR were predicted [42] and experimentally confirmed [27,28], and the Kerr-like cSPM-RR was studied numerically [15].

For a physical interpretation, it is instructive to transform the interaction back to the lab-frame coordinate by using the soliton dispersion relation, $k_{s}(\omega)=k\left(\omega_{s}\right)+\left(\omega-\omega_{s}\right) /$ $v_{g, s}+q_{s}$. In the SPM-RR case, we immediately get the well-known dispersion relation, $k\left(\omega_{\mathrm{RR}}\right)=k_{s}\left(\omega_{\mathrm{RR}}\right)$ [41], caused by self-acting 4WM (i.e., "degenerate" [41] 4WM). In the SFG case (the $A^{2}$ term), the rewritten phase-matching condition is

$k\left(\omega_{\mathrm{RR}}\right)=2 k_{s}\left(\omega_{\mathrm{RR}}\right)-\omega_{\mathrm{RR}} / v_{g, s}=k_{s}\left(\omega_{a}\right)+k_{s}\left(\omega_{b}\right)$,

i.e., SFG between soliton photons at two different frequencies and where energy conservation dictates $\omega_{a}+\omega_{b}=$ $\omega_{\mathrm{RR}}$. If we take $\omega_{a}=\omega_{s}$, then $\omega_{b}=\omega_{\mathrm{RR}}-\omega_{s}$. We now show how the second-harmonic generation (SHG) phase-mismatch parameter $\Delta k_{\mathrm{SHG}}$ is affecting this phasematching condition: by expanding the dispersion operator in Eq. (5) around the second-harmonic (SH) frequency of the soliton frequency, $\omega_{2}=2 \omega_{s}$, we get

$$
\tilde{D}_{2 \omega_{s}}\left(\omega_{\mathrm{RR}}\right)-\left(\omega_{\mathrm{RR}}-2 \omega_{s}\right) d_{\mathrm{SHG}}^{\mathrm{GVM}}+\Delta k_{\mathrm{SHG}}=2 q_{s},
$$

where $d_{\mathrm{SHG}}^{\mathrm{GVM}}=k_{1}\left(\omega_{s}\right)-k_{1}\left(2 \omega_{s}\right)$ is the group-velocity mismatch coefficient between the soliton and its SH. Clearly, the SHG phase-mismatch parameter, $\Delta k_{\mathrm{SHG}}=$ $k\left(2 \omega_{s}\right)-2 k\left(\omega_{s}\right)$, allows for a tunable control over the spectral position of the RR wave. In a birefingent critically phase-mismatched interaction, $\Delta k_{\mathrm{SHG}}$ can be tuned by the crystal angle, while in a noncritical phase-mismatched interaction (e.g., PPLN), $\Delta k_{\mathrm{SHG}}$ is effectively controlled by the poling pitch. Interestingly, Eq. (8) is identical to the SH nonlocal resonance condition $[43,44]$ that recently was confirmed experimentally [45]. Thus, in the defocusing soliton parameter range, the tunable nonlocal $\mathrm{SH}$ wave in [45] is identical in nature to the SFG-RR wave we predict here. Additionally, the simulations in [30] seem also to show an SFG-RR wave. However, we stress that these previous observations did not identify these peaks as resonant radiation.

The DFG case can similarly be written as

$$
k\left(\omega_{\mathrm{RR}}\right)=\omega_{\mathrm{RR}} / v_{g, s}=k_{s}\left(\omega_{a}\right)+k_{s}\left(\omega_{b}\right),
$$

where $\omega_{a}-\omega_{b}=\omega_{\mathrm{RR}}$. Thus, the physics behind this condition is DFG between soliton photons at different frequencies, and it explains why the soliton wave number $q_{s}$ is absent in Eq. (6). Expanding this phase-matching condition around the DFG frequency reveals a relation similar to the SFG case, 
namely that the DFG phase mismatch can tune the RR spectral position. The DFG-RR phase-matching condition is not easy to fulfill. To see that, Eq. (6) is expressed as

$$
v_{\mathrm{ph}}\left(\omega_{\mathrm{RR}}\right)=v_{g, s},
$$

i.e., that the RR phase velocity $v_{\mathrm{ph}}\left(\omega_{\mathrm{RR}}\right)$ is the same as the soliton group velocity. This is equivalent to the so-called velocity-matching condition, encountered, e.g., when generating $\mathrm{THz}$ radiation in a quadratic nonlinear crystal through DFG [46]. The intuitive explanation behind the $\mathrm{THz}$ velocity-matching condition is that the $\mathrm{THz}$ carrier wave relies on the different colors of the pump wave having the same group velocity, i.e., that they move as a single wave packet. Essentially the same can be said about the soliton in the DFG-RR case because due to the straight-line dispersion of the soliton, its photons will move with the same group velocity no matter at what frequency they are taken from. Matching the phase- and group velocities is not easy, especially because the DFG process implies that the converted photon is at a lower frequency than the pump photons. This makes velocity matching virtually impossible, except when the converted wave lies beyond an IR resonance (like the $\mathrm{THz}$ case) where the drawback is a very low yield.

However, by exploiting quasiphase matching (QPM), we can achieve velocity matching in the same transparency window as the soliton, and thereby observe the DFG-RR for the first time. Taking a square-grating periodic-poling structure of the quadratic nonlinearity with pitch $\Lambda$, the $3 \mathrm{WM}$ conditions change to

$$
\begin{aligned}
& \tilde{D}_{\omega_{s}}\left(\omega_{\mathrm{RR}}\right)=2 q_{s}-\Delta_{\mathrm{pg}}+k_{\Lambda}, \quad\left(\mathrm{SFG}-\mathrm{RR}, A^{2}\right), \\
& \tilde{D}_{\omega_{s}}\left(\omega_{\mathrm{RR}}\right)=\Delta_{\mathrm{pg}}-k_{\Lambda}, \quad\left(\mathrm{DFG}-\mathrm{RR}, A^{*} A\right) .
\end{aligned}
$$

We see that by tuning the QPM wave number, $k_{\Lambda}=2 \pi / \Lambda$, we can now manipulate the phase-matching conditions and get tunable control over the RR frequency.

The soliton we excite here is a bright self-defocusing temporal soliton. The negative (self-defocusing) nonlinearity is created through strongly phase-mismatched (i.e., cascaded) second-harmonic generation (SHG). Essentially, the pump wave will experience a Kerr-like nonlinear refractive index, $n_{2, \text { casc }} \propto-\left(\chi^{(2)}\right)^{2} / \Delta k_{\text {SHG }}$ [19], and this will compete with the intrinsic material self-focusing Kerr nonlinearity, $n_{2, \text { Kerr }} \propto \chi^{(3)}$. If the SHG phase mismatch $\Delta k_{\mathrm{SHG}}$ is made suitably small and the residual effective nonlinear refractive index, $n_{2, \text { eff }}=n_{2, \text { casc }}+n_{2, \text { Kerr }}$, becomes negative, the soliton can be excited in the normal group-velocity dispersion $(\mathrm{GVD})$ regime $\left[k_{2}\left(\omega_{1}\right)>0\right]$ below the ZDW.

The experimental setup was similar to [27], and consisted of only the pump, a silver-mirror telescope, and the PPLN crystal. The pump laser was a $1 \mathrm{kHz}$ OPA system, and wavelengths $1.55-1.85 \mu \mathrm{m}$ were used, all located below the ZDW of LN. The pump pulse duration was $60 \mathrm{fs}$ and close to the transform limit and was loosely collimated before the crystal ( $0.5 \mathrm{~mm}$ FWHM spot size). Several bulk PPLN crystals with multigrating structures were used with pitch gratings from $\Lambda=27.0-31.6 \mu \mathrm{m}$, all designed to exploit the large $d_{33}$ quadratic nonlinearity; in this range, $\left|n_{2, \text { casc }}\right| / n_{2, \text { Kerr }} \simeq 1.5-2.0$. The spectrum was measured in the mid IR $(\lambda>2.3 \mu \mathrm{m})$, with an FPAS-1600 spectrometer (Infrared Systems) with a cooled MCT detector, and long-pass filters were used to selectively cover the $2-6 \mu \mathrm{m}$ range. In the visible and near-IR range, compact spectrometers were used, based in $\mathrm{Si}$ and InGaAs CCD detectors, respectively.

Figure 1 shows a typical high-intensity spectrum. The pump pulse (80 nm FWHM) has experienced massive broadening, and a supercontinuum is formed spanning over 3 octaves $(550-5000 \mathrm{~nm})$. The soliton has clearly broadened to the blue: a "center-of-mass" calculation gave $\lambda_{s}=1.68 \mu \mathrm{m}$, which was then used to calculate the RR phase-matching curves from the expressions derived above. The soliton wave number $q_{s}$ was estimated to be that of a $T_{s}=10 \mathrm{fs}$ soliton (a typical value from simulations); assuming that such a soliton will have unity soliton order, one can use the expression for $q_{s}=n_{2, \text { eff }} I_{s} \omega_{s} /(2 c)$ [42] and that of the effective soliton order [47] to get $q_{s}=-2 k_{2}\left(\omega_{s}\right) / T_{s}^{2}$. By comparing these curves with the experimental data, we identify a number of RR peaks: firstly, the broad mid-IR peak above the ZDW is the Kerr SPM-RR wave, identical in nature to the recent observations in other crystals $[27,28]$. Secondly, a peak is located at $1002 \mathrm{~nm}$. Even if this is close to a QPM phase-matching line (dashed line), we show below evidence that this is indeed the SFG-RR wave. Finally, the peak at $4700 \mathrm{~nm}$ is the DFG-RR wave. In [22-24], mid-IR spectral peaks were also observed, but by carrying out a similar phase-matching analysis as above, we can only conclude that these were SPM-RR waves. In the low-wavelength range, there are too many gaps to form a continuum. Many narrow lines were seen stemming from QPM higher-order resonances, e.g., the SHG QPM conditions, $k(\omega)-2 k(\omega / 2)-m_{0} k_{\Lambda}=0$, with $m_{0}$ odd. The plot also shows the result of a numerical simulation [33], showing excellent quantitative agreement. Finally, we mention that the elusive cSPM-RR and THG-RR waves were not observed.

Figure 2 shows how the spectrum changes with intensity. Based on the appearance of the DWs, we estimate that the soliton forms for much lower intensities (at around $50 \mathrm{GW} / \mathrm{cm}^{2}$ ) than in unpoled LN [26,27], which is due to the larger effective nonlinearity as QPM significantly reduces the SHG phase mismatch. For increasing intensities, the soliton becomes more blueshifted (the black dashed line shows the calculated "center-of-mass" soliton wavelength). This is in stark contrast to the massive Raman-induced redshift observed in unpoled LN [26,27] and is a consequence of pumping close to the ZDW, which makes the soliton recoil towards the blue. The blueshifted soliton wavelength directly affects all three phase-matching 


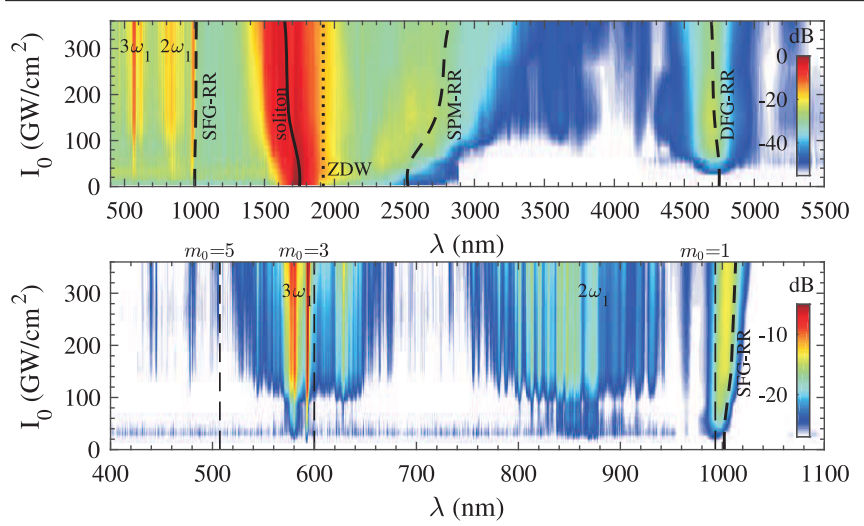

FIG. 2. False-color representation of the experimental supercontinua for various intensities with the same parameters as Fig. 1. Dashed lines: theoretical phase-matching wavelengths using the extracted soliton wavelengths (black line). Bottom: details of the visible and short-wavelength near-IR range, including calculated QPM resonances.

conditions. This blueshift explains why the SPM-RR plateau redshifts with increasing intensity. The DFG-RR phase-matched RR wave remains more or less constant, but the SFG-RR wave noticeably changes wavelength from low to high intensity as the soliton blue shifts; see bottom plot. Even if this peak lies quite close to the $m_{0}=1 \mathrm{QPM}$ line, there is evidence that it is indeed a DW: it clearly follows the calculated SFG-RR phase matching as the intensity increases and it is also too broadband to be a QPM line; note in contrast how narrow the $m_{0}=3$ and 5 QPM lines are. For high intensities, the SPM-RR plateau flattens, and numerical simulations [33] indicate that this is due to increased pump depletion as well as significant self-steepening during the soliton formation stage. We are currently investigating this further. The visible range contains spectral "copies" of the soliton supercontinuum at the harmonic wavelengths $\left(2 \omega_{1}, 3 \omega_{1}\right)$; this is due to trapped radiation caused by the cascaded (i.e., phasemismatched) nonlinearities [44,45,48], giving a coherent extension of the supercontinuum into the visible. The simulations indeed confirmed that the supercontinua had a high degree of coherence, also in the trapped harmonic extensions.

The parametric tunability of the TWM nonlinearities gives a mid-IR edge of the supercontinuum output that is tunable, which is demonstrated in Fig. 3(a), where the poling pitch is varied. Note also the excellent agreement with the theoretical phase-matching calculations (see zoom in the inset). The theoretical curves also show the further potential in tuning the $3 \mathrm{WM}$ RR waves: essentially, the whole near- and mid-IR transparency range of LN is covered, although practically the soliton and the SPM-RR will dominate the 1.5-3.5 $\mu \mathrm{m}$ range. The plot in Fig. 3(b) confirms that indeed a broader tuning range in the mid IR is possible: the DFG-RR waves were recorded in a $20 \mathrm{~mm}$ long PPLN crystal with 10 different QPM pitch values.
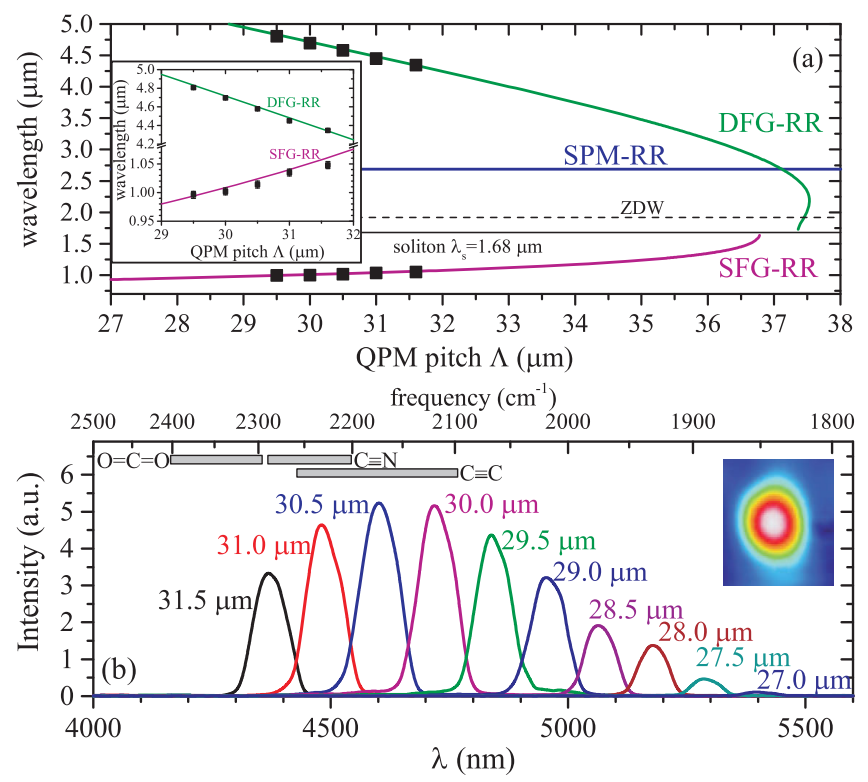

FIG. 3. (a) Calculated tuning curves for SPM, DFG, and SFG RR waves (using a fixed $\lambda_{s}=1.68 \mu \mathrm{m}$ ), plotted with data from the same experiment as Fig. 1, but where the QPM pitch was varied. Inset: details around the experimental data. (b) Experimental mid-IR spectra showing the DFG-RR peaks, recorded with a $20 \mathrm{~mm}$ PPLN with 10 different QPM pitch values, using $\lambda_{0}=1.75 \mu \mathrm{m}$ and $I_{0}=55 \mathrm{GW} / \mathrm{cm}^{2}$; note the linear $y$ axis. Inset: typical transverse beam profile of the long-pass filtered mid-IR RR wave.

All spectra were recorded one after another so the intensity magnitudes are therefore absolute and can be related to each other. The tuning range demonstrated here has a quite specific importance: as the inset bars indicate, the IR molecular vibration absorption bands that are present in this range are IR stretching modes, which, apart from the wellknown band for $\mathrm{CO}_{2}$ in the gas phase, include the important alkyne and nitrile stretching modes. This degree of tunability is quite unique, and it could be exploited by using an adiabatic change in the pitch along the crystal to give a broader and smoother DFG-RR peak to cover the spectral gap towards the SPM-RR. In the 4WM case, the RR position has little or no tunability except in a gas-filled hollow-core fiber, where the pressure may control both the dispersion and the nonlinearity [6-9]. However, it also requires adjusting the pump power.

Similar to [27], we isolated the mid-IR DFG-RR waves with a long-pass filter and measured them to be subpicosecond pulses with a significant amount of chirp (this is expected as the RR waves are inherently dispersive). Using a $\mathrm{THz}$ camera, we measured the isolated mid-IR beam profiles, revealing Gaussian-like shapes [Fig. 3(b), inset].

In conclusion, we have shown soliton-induced resonant radiation mediated by $\chi^{(2)}$ three-wave mixing nonlinear terms representing sum- and difference-frequency generation $\left(A^{2}\right.$ and $A^{*} A$, respectively). These provide a powerful extension of the well-known resonant radiation induced by 
$\chi^{(3)}$ four-wave mixing, in particular due to the broadband parametric tunability of the resonant wavelengths provided by the SFG or DFG phase-mismatch parameters. Our experiment was conducted in PPLN crystals, where the parametric tunability came from changing the QPM pitch. This, combined with the excitation of a self-defocusing soliton, allowed us to phase match the DFG resonant radiation wave in the mid IR. The SFG and SPM resonant radiation waves were also observed and the full supercontinuum spanned over 3 octaves (550-5000 nm). Our results could find direct use in soliton-based frequencycomb generation in microresonators [49-51], for which quadratic nonlinear materials are currently being explored for on-chip inherent harmonic conversion of the IR comb lines [52-56]. Considering that the standard cavity nonlinear model [55] was recently adopted for the quadratic nonlinearity case [57], which showed similar nonlinear terms as in Eq. (1), we believe that the demonstrated SFG and DFG resonant radiation waves can provide a unique tunable control over the coherent extension of the comb lines.

Support from the Danish Council for Independent Research (Grant no. 11-106702) is acknowledged. X. L. Zeng acknowledges the support of National Natural Science Foundation of China (11274224) and from FP7-Marie Curie Actions (Grant no. PIIF-GA-2009-253289). M. B. acknowledges fruitful discussions with Peter Uhd Jepsen.

*Corresponding author.

moba@fotonik.dtu.dk.

†Present address: École Polytechnique Fédérale de Lausanne, CH-1015 Lausanne, Switzerland.

"Permanent address: Key Laboratory of Special Fiber Optics and Optical Access Networks, Shanghai University, Shanghai 200072, China.

[1] L. F. Mollenauer, R. H. Stolen, and J. P. Gordon, Experimental Observation of Picosecond Pulse Narrowing and Solitons in Optical Fibers, Phys. Rev. Lett. 45, 1095 (1980).

[2] P. K. A. Wai, C. R. Menyuk, Y. C. Lee, and H.H. Chen, Nonlinear pulse propagation in the neighborhood of the zero-dispersion wavelength of monomode optical fibers, Opt. Lett. 11, 464 (1986).

[3] N. Akhmediev and M. Karlsson, Cherenkov radiation emitted by solitons in optical fibers, Phys. Rev. A 51, 2602 (1995).

[4] D. V. Skryabin and A. V. Gorbach, Colloquium: Looking at a soliton through the prism of optical supercontinuum, Rev. Mod. Phys. 82, 1287 (2010).

[5] J. M. Dudley, G. Genty, and S. Coen, Supercontinuum generation in photonic crystal fiber, Rev. Mod. Phys. 78, 1135 (2006).

[6] N. Y. Joly, J. Nold, W. Chang, P. Hölzer, A. Nazarkin, G. K. L. Wong, F. Biancalana, and P. S. J. Russell, Bright Spatially Coherent Wavelength-Tunable Deep-UV Laser Source Using an Ar-Filled Photonic Crystal Fiber, Phys. Rev. Lett. 106, 203901 (2011).
[7] K. F. Mak, J. C. Travers, P. Hölzer, N. Y. Joly, and P. S. J. Russell, Tunable vacuum-UV to visible ultrafast pulse source based on gas-filled kagome-PCF, Opt. Express 21, 10942 (2013).

[8] F. Belli, A. Abdolvand, W. Chang, J.C. Travers, and P. S. Russell, Vacuum-ultraviolet to infrared supercontinuum in hydrogen-filled photonic crystal fiber, Optica 2, 292 (2015).

[9] A. Ermolov, K. F. Mak, M. H. Frosz, J.C. Travers, and P. S. J. Russell, Supercontinuum generation in the vacuum ultraviolet through dispersive-wave and soliton-plasma interaction in a noble-gas-filled hollow-core photonic crystal fiber, Phys. Rev. A 92, 033821 (2015).

[10] P. Beaud, W. Hodel, B. Zysset, and H. Weber, Ultrashort pulse propagation, pulse breakup, and fundamental soliton formation in a single-mode optical fiber, IEEE J. Quantum Electron. 23, 1938 (1987).

[11] F. W. Wise, I. A. Walmsley, and C. L. Tang, Simultaneous formation of solitons and dispersive waves in a femtosecond ring dye laser, Opt. Lett. 13, 129 (1988).

[12] A. S. Gouveia-Neto, M. E. Faldon, and J. R. Taylor, Solitons in the region of the minimum group-velocity dispersion of single-mode optical fibers, Opt. Lett. 13, 770 (1988).

[13] E. Rubino, J. McLenaghan, S. C. Kehr, F. Belgiorno, D. Townsend, S. Rohr, C. E. Kuklewicz, U. Leonhardt, F. König, and D. Faccio, Negative-Frequency Resonant Radiation, Phys. Rev. Lett. 108, 253901 (2012).

[14] M. Petev, N. Westerberg, D. Moss, E. Rubino, C. Rimoldi, S. L. Cacciatori, F. Belgiorno, and D. Faccio, Blackbody Emission from Light Interacting with an Effective Moving Dispersive Medium, Phys. Rev. Lett. 111, 043902 (2013).

[15] M. Conforti, N. Westerberg, F. Baronio, S. Trillo, and D. Faccio, Negative-frequency dispersive wave generation in quadratic media, Phys. Rev. A 88, 013829 (2013).

[16] E. Rubino, A. Lotti, F. Belgiorno, S. L. Cacciatori, A. Couairon, U. Leonhardt, and D. Faccio, Soliton-induced relativisticscattering and amplification, Sci. Rep. 2, 932 (2012).

[17] M. Conforti, A. Marini, T.X. Tran, D. Faccio, and F. Biancalana, Interaction between optical fields and their conjugates in nonlinear media, Opt. Express 21, 31239 (2013).

[18] C. R. Lourés, D. Faccio, and F. Biancalana, Nonlinear Cavity and Frequency Comb Radiations Induced by Negative Frequency Field Effects, Phys. Rev. Lett. 115, 193904 (2015).

[19] R. DeSalvo, D. Hagan, M. Sheik-Bahae, G. Stegeman, E. W. Van Stryland, and H. Vanherzeele, Self-focusing and self-defocusing by cascaded second-order effects in KTP, Opt. Lett. 17, 28 (1992).

[20] X. Liu, L.-J. Qian, and F.W. Wise, High-energy pulse compression by use of negative phase shifts produced by the cascaded $\chi^{(2)}: \chi^{(2)}$ nonlinearity, Opt. Lett. 24, 1777 (1999).

[21] S. Ashihara, J. Nishina, T. Shimura, and K. Kuroda, Soliton compression of femtosecond pulses in quadratic media, J. Opt. Soc. Am. B 19, 2505 (2002).

[22] C. Langrock, M. M. Fejer, I. Hartl, and M. E. Fermann, Generation of octave-spanning spectra inside reverseproton-exchanged periodically poled lithium niobate waveguides, Opt. Lett. 32, 2478 (2007). 
[23] C. R. Phillips, C. Langrock, J. S. Pelc, M. M. Fejer, J. Jiang, M. E. Fermann, and I. Hartl, Supercontinuum generation in quasi-phase-matched $\mathrm{LiNbO}_{3}$ waveguide pumped by a Tm-doped fiber laser system, Opt. Lett. 36, 3912 (2011).

[24] C. R. Phillips, C. Langrock, J. S. Pelc, M. M. Fejer, I. Hartl, and M. E. Fermann, Supercontinuum generation in quasiphasematched waveguides, Opt. Express 19, 18754 (2011).

[25] H. R. Guo, B. B. Zhou, M. Steinert, F. Setzpfandt, T. Pertsch, H. P. Chung, Y. H. Chen, and M. Bache, Supercontinuum generation in quadratic nonlinear waveguides without quasi-phase matching, Opt. Lett. 40, 629 (2015).

[26] B. B. Zhou, A. Chong, F. W. Wise, and M. Bache, Ultrafast and Octave-Spanning Optical Nonlinearities from Strongly Phase-Mismatched Quadratic Interactions, Phys. Rev. Lett. 109, 043902 (2012).

[27] B. Zhou, H. Guo, and M. Bache, Energetic mid-IR femtosecond pulse generation by self-defocusing soliton-induced dispersive waves in a bulk quadratic nonlinear crystal, Opt. Express 23, 6924 (2015).

[28] B. Zhou and M. Bache, Dispersive waves induced by selfdefocusing temporal solitons in a beta-barium-borate crystal, Opt. Lett. 40, 4257 (2015).

[29] B. Zhou and M. Bache, Invited article: Multiple-octave spanning high-energy mid-IR supercontinuum generation in bulk quadratic nonlinear crystals, APL Photonics 1, 050802 (2016).

[30] M. Conforti, F. Baronio, and C. De Angelis, Nonlinear envelope equation for broadband optical pulses in quadratic media, Phys. Rev. A 81, 053841 (2010).

[31] M. Bache, The nonlinear analytical envelope equation in quadratic nonlinear crystals, arXiv:1603.00188.

[32] F. Baronio, M. Conforti, C. D. Angelis, D. Modotto, S. Wabnitz, M. Andreana, A. Tonello, P. Leproux, and V. Couderc, Second and third order susceptibilities mixing for supercontinuum generation and shaping, Opt. Fiber Technol. 18, 283 (2012).

[33] See Supplemental Material http://link.aps.org/supplemental/ 10.1103/PhysRevLett.118.143901, which includes Refs. [34-40], for a complete description of the envelope equation, the derivation of the phase matching conditions and discussion of how quasiphase matching is introduced, and additional numerical simulation examples.

[34] M. Conforti, F. Baronio, and C. De Angelis, Ultrabroadband optical phenomena in quadratic nonlinear media, IEEE Photonics J. 2, 600 (2010).

[35] H. Guo, X. Zeng, B. Zhou, and M. Bache, Nonlinear wave equation in frequency domain: accurate modeling of ultrafast interaction in anisotropic nonlinear media, J. Opt. Soc. Am. B 30, 494 (2013).

[36] O. Gayer, Z. Sacks, E. Galun, and A. Arie, Temperature and wavelength dependent refractive index equations for $\mathrm{MgO}$ doped congruent and stoichiometric $\mathrm{LiNbO}_{3}$, Appl. Phys. B 91, 343 (2008).

[37] Y.-S. Lee, T. Meade, V. Perlin, H. Winful, T. B. Norris, and A. Galvanauskas, Generation of narrow-band terahertz radiation via optical rectification of femtosecond pulses in periodically poled lithium niobate, Appl. Phys. Lett. 76, 2505 (2000).

[38] J. L'huillier, G. Torosyan, M. Theuer, Y. Avetisyan, and R. Beigang, Generation of $\mathrm{THz}$ radiation using bulk, periodically and aperiodically poled lithium niobate-part 1: Theory, Appl. Phys. B 86, 185 (2007).

[39] J. L'huillier, G. Torosyan, M. Theuer, C. Rau, Y. Avetisyan, and R. Beigang, Generation of $\mathrm{THz}$ radiation using bulk, periodically and aperiodically poled lithium niobate-part 2: Experiments, Appl. Phys. B 86, 197 (2007).

[40] I. Shoji, T. Kondo, A. Kitamoto, M. Shirane, and R. Ito, Absolute scale of second-order nonlinear-optical coefficients, J. Opt. Soc. Am. B 14, 2268 (1997).

[41] D. V. Skryabin and A. V. Yulin, Theory of generation of new frequencies by mixing of solitons and dispersive waves in optical fibers, Phys. Rev. E 72, 016619 (2005).

[42] M. Bache, O. Bang, B. B. Zhou, J. Moses, and F. W. Wise, Optical Cherenkov radiation in ultrafast cascaded secondharmonic generation, Phys. Rev. A 82, 063806 (2010).

[43] M. Bache, O. Bang, J. Moses, and F. W. Wise, Nonlocal explanation of stationary and nonstationary regimes in cascaded soliton pulse compression, Opt. Lett. 32, 2490 (2007).

[44] M. Bache, O. Bang, W. Krolikowski, J. Moses, and F. W. Wise, Limits to compression with cascaded quadratic soliton compressors, Opt. Express 16, 3273 (2008).

[45] B. Zhou, H. Guo, and M. Bache, Soliton-induced nonlocal resonances observed through high-intensity tunable spectrally compressed second-harmonic peaks, Phys. Rev. A 90, 013823 (2014).

[46] A. Nahata, A. S. Weling, and T. F. Heinz, A wideband coherent terahertz spectroscopy system using optical rectification and electro-optic sampling, Appl. Phys. Lett. 69, 2321 (1996).

[47] M. Bache, J. Moses, and F. W. Wise, Scaling laws for soliton pulse compression by cascaded quadratic nonlinearities, J. Opt. Soc. Am. B 24, 2752 (2007); Erratum, J. Opt. Soc. Am. B 27, 2505(E) (2010).

[48] G. Valiulis, V. Jukna, O. Jedrkiewicz, M. Clerici, E. Rubino, and P. DiTrapani, Propagation dynamics and X-pulse formation in phase-mismatched second-harmonic generation, Phys. Rev. A 83, 043834 (2011).

[49] P. Del'Haye, A. Schliesser, O. Arcizet, T. Wilken, R. Holzwarth, and T. J. Kippenberg, Optical frequency comb generation from a monolithic microresonator, Nature (London) 450, 1214 (2007).

[50] T. Herr, V. Brasch, J. D. Jost, Y. C. Wang, M. N. Kondratiev, L. M. Gorodetsky, and T. J. Kippenberg, Temporal solitons in optical microresonators, Nat. Photonics 8, 145 (2014).

[51] V. Brasch, M. Geiselmann, T. Herr, G. Lihachev, M. H. P. Pfeiffer, M. L. Gorodetsky, and T. J. Kippenberg, Photonic chip-based optical frequency comb using soliton Cherenkov radiation, Science 351, 357 (2016).

[52] H. Jung, R. Stoll, X. Guo, D. Fischer, and H. X. Tang, Green, red, and IR frequency comb line generation from single IR pump in AIN microring resonator, Optica 1, 396 (2014).

[53] S. Miller, K. Luke, Y. Okawachi, J. Cardenas, A. L. Gaeta, and M. Lipson, On-chip frequency comb generation at visible wavelengths via simultaneous second- and thirdorder optical nonlinearities, Opt. Express 22, 26517 (2014).

[54] I. Ricciardi, S. Mosca, M. Parisi, P. Maddaloni, L. Santamaria, P. De Natale, and M. De Rosa, Frequency comb generation in quadratic nonlinear media, Phys. Rev. A 91, 063839 (2015). 
[55] F. Leo, T. Hansson, I. Ricciardi, M. De Rosa, S. Coen, S. Wabnitz, and M. Erkintalo, Walk-Off-Induced Modulation Instability, Temporal Pattern Formation, and Frequency Comb Generation in Cavity-Enhanced Second-Harmonic Generation, Phys. Rev. Lett. 116, 033901 (2016).

[56] H. Jung, X. Guo, N. Zhu, S. B. Papp, S. A. Diddams, and H.X. Tang, Phase-dependent interference between frequency doubled comb lines in a $\chi^{(2)}$ phase-matched aluminum nitride microring, Opt. Lett. 41, 3747 (2016).

[57] T. Hansson, F. Leo, M. Erkintalo, J. Anthony, S. Coen, I. Ricciardi, M. D. Rosa, and S. Wabnitz, Single envelope equation modeling of multi-octave comb arrays in microresonators with quadratic and cubic nonlinearities, J. Opt. Soc. Am. B 33, 1207 (2016). 